\title{
Statements Concerning the Patellofemoral Joint
}

\author{
Lars Blønd \\ Department of Orthopedic Surgery, The Zealand University Hospital, Koege, Denmark
}

*Corresponding author: Lars Blønd, Department of Orthopedic Surgery, The Zealand University Hospital, Koege, Lykkebaekvej 1, DK-4600

\begin{abstract}
This is different statements in respect to understanding and treating patients having patellofemoral problems. The statements are based upon scientific data and clinical experience and they reflect the authors opinion. The statements are specifically selected in relation to specific topics with little consensus and/or frequent misunderstandings. Subjects like first time patella dislocations, trochlea anatomy, tibia tubercle trochlea groove distance (TTTG), patella alta, medial patellofemoral ligament reconstructions, trochlear dysplasia, trochleoplasty, anterior knee pain and Hoffa fat pad impingement, will briefly be commented on.
\end{abstract}

Keywords: Patellofemoral; Patella instability; Radiologic; Trochlear Dysplasia; Patella Alta; Anterior Knee Pain

\section{Introduction}

This is a list of statements in respect to understanding and treating patients having patellofemoral symptoms. Some of these statements might be controversial and disruptive in respect to current understandings. The statements are too high degree based upon scientific literature, but they also reflect 25 years of clinical and surgical experience dealing with patellofemoral patients.

\section{First time patella dislocation (FTPD)}

Some first FTP dislocators continue to be trouble by instability symptoms, despite they do not redislocate, and they need surgical stabilisation [1]. Two recent metaanalysis demonstrates that relative to conservative treatment, is the outcome of patella stablising surgery favorable, both in respect to risk of redislocation, but also in respect to clinical outcome [2,3]. However you can always prescribe physiotherapy, even so there is still absence of evidence, that this reduce patella instability symptoms. In respect to using a brace after FTPD, did a recent study with more than 600 patients, not find any positive effect of immobilization in a brace, and moreover did see any reduced risk of redislocation for those who had undergone physiotherapy [4]. In case of surgery is decided this have to based on meny a la carte, correcting important anatomic predisposing factors.

\section{Trochlea anatomy}

Of outmost importance and often overlooked, is when you evaluate the PF joint trochlear configuration, since this change from being more shallow proximal to becoming more deep when going distally. See Figure 1. Numerous scientific papers do not take this key issue into account, and this is a broad problem when reports on different measures that were meant to objectively outline the configuration of the trochlear configuration, are inaccurate. For example, two measures like the sulcus angle and the lateral trochlear inclination angle (LTI) varies substantially from proximal to distal. Studies regularly report on data, where the measures have been obtained relative distally and this has caused discrepancies. Typically, these studies aim to find specific anatomic differences characterizing patients having patellar instability or anterior knee pain (AKP), by a comparison to a group of normal knees. Thereby they overlook significant differences due to the above mentioned problem. These misconceptions are regrettable repeated when later metanalysis are accomplished. These mistakes have led to scientific frauds, like absence in studies searching for a correlation between AKP and anatomy.

\section{Tibial tubercle trochlear grove (TTTG)}

What is a normal TTTG distance and what is abnormal? Tan el al. reported that the threshold or cut-off for abnormality is different from MRI and CT scan [5]. In a metanalysis they found the cut off for an abnormal TTTG distance to be less than the often reported $20 \mathrm{~mm}$. When measured on MRI, the mean TT-TG distance for the control group was $9.83 \mathrm{~mm}$ (95\% CI: 9.11-10.54), while the mean TT-TG distance for patients with patellofemoral instability was $15.33 \mathrm{~mm}$ (95\% CI: 14.24-16.42). What also have to be taken into account when you evaluate the TTTG distance, is also to correct for the patient's height [6]. Noteworthy is that new unpublished 
data (personal message) demonstrate, that an increased TTTG distance, in generally speaking is due to a medialized trochlear groove (TG) and not an externally placed tibial tubercle (TT). This means that the TT-TG to a higher degree reflects trochlear asymmetry. Furthermore, an often claimed misunderstanding is that the TT-TG distance is correlated to the more clinical $Q$ angle, but inconsistency has been highlighted [7]. In conclusion is the $\mathrm{Q}$ angle a non-reproducible measure.
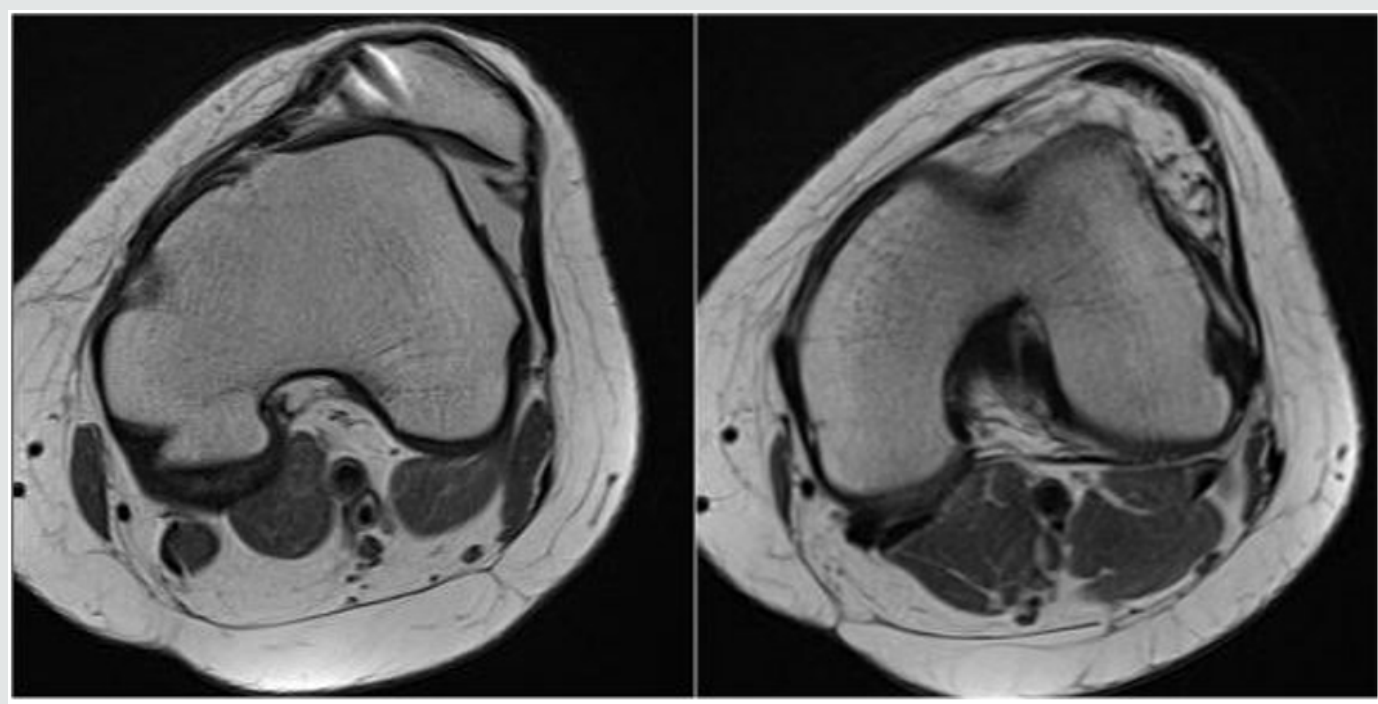

Figure 1: This figure illustrates the different trochlea configuration in the same knee with trochlear dysplasia, from proximal to distal. This patient has trochlear dysplasia and have had an MPFL reconstruction that failed.

\section{Patella alta and radiologic indexes}

The second most important factor for patellofemoral malalignment is Patella Alta and new consensus among Patellofemoral specialist are gradually evolving. Patella height should not be correlated to the length of the patella ligament and neither to the patella's relation to the tibia. What is of interest is the patella engagement into the trochlear groove and the overlap between the patella cartilage and trochlea cartilage surfaces. This relationship depends on the patella configuration and the length of the trochlear. The Patellotrochlear index from Biedert and Albrecht and the later Sagittal Patellofemoral Engagement index measure this engagement [8,9]. Some are still critical about this statement, since those indexes are sensitive to the angle of knee flexion. These indexes are not as reproducible as older measures, as for instance the Insall-Salvati and Caton-Dechamps indexes. However, to measure something irrelevant is a fault and just because old indexes a more reliable, this does not qualify them to be more relevant. Most noteworthy does these old indexes not account for the very variable length of the trochlear. It becomes logic that when the patella cartilage and trochlear cartilage overlaps is a minor figure, measuring only a few millimeters, it by itself becomes less reproducible. See Figure 2. However, with everything said those engagement indexes, are even so the most precise to express the patella height [10]. To understand above mentioned is of almost importance, not only for science, but also in daily clinic settings. My claim is, that when having overlooked those engagement indexes, and instead have used old indexes, this had led to a number of failed surgeries. Number of destabilizations of the distal tubercle, have resulted in a Patella Baja situation, mistakenly interpreted as arthrofibrosis and caused negative outcomes with pain and flexion defects for those affected. In the other end of the spectrum, have lengthening of patella ligaments, due to erroneous assessments mistakenly concluding it was Patella Baja, lead to false surgeries. When it comes in terms to surgical treatment of Patella Alta by distalisation of the tibial tubercle, there have been some controversies. A prevailing misunderstanding is that the knee cartilage in the patellofemoral joint will be overloaded, due to an increased traction forces, when the patella is pulled down. Actually, this is precisely opposite, since the cartilage overlap increase and the pressure per square meter of cartilage will diminish [11].

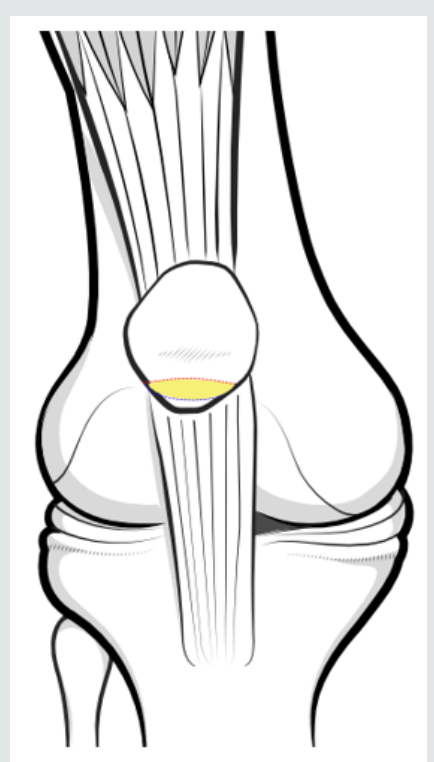

Figure 2: This figure illustrates the overlap between patella and trochlea cartilage in full extension. In Patella Alta this overlap is with a minor area causing increased pressure per square meter of cartilage. 


\section{Medial patellofemoral ligament reconstruction MPFL-R}

In some clinical settings have MPFL-R become a panacea for all patella stabilizing surgeries. This might be a reflection that numerous papers have demonstrated favorable results after MPFL reconstructions. However, most of these papers are with short time follow-up. Papers generally fails to include patients having more pronounced underlying pathomorphology when it comes to longer follow-up studies. Increasing amount of evidence demonstrate that MPFL reconstructions might stabilize most unstable patellae for a period, however if you omit correct the underlining biomechanical problem, the obtained stability is likely to be temporary and the clinical outcome will not only be suboptimal but also deteriorate over time [12,13]. Feucht MJ, Mehl J, Forkel P, Achtnich A, Schmitt A, Izadpanah K, Imhoff AB, Berthold DP (2020) Failure Analysis in Patients With Patellar Redislocation After Primary Isolated Medial Patellofemoral Ligament Reconstruction. Orthop J Sports Med 8:110 Also noteworthy was the metanalysis from Balcerek et al. [14] who found that the risk of patella re-dislocation was diminished if trochleoplasty plus extensor balancing procedure was done, compared to those having only isolated MPFL reconstructions.

\section{Trochlear dysplasia}

Still there is no consensus in respect to define Trochlear Dysplasia. The classic Dejour classification is based upon x-rays and the inter and intra observer variation have been found unreliable [15-18]. Kazley J, Banerjee S (2019) Classifications in Brief: The Dejour Classification of Trochlear Dysplasia. Clin Orthop Relat Res 477:2380-2386

Koëter S, Bongers EMHF, de Rooij J, van Kampen A (2006) Minimal rotation aberrations cause radiographic misdiagnosis of trochlear dysplasia. Knee Surg Sports Traumatol Arthrosc 14:713-7

Rémy F, Chantelot C, Fontaine C, Demondion X, Migaud H, Gougeon F (1998) Inter- and intraobserver reproducibility in radiographic diagnosis and classification of femoral trochlear dysplasia. Surg Radiol Anat Surg Radiol Anat 20:285-9

Stepanovich M, Bomar JD, Pennock AT (2016) Are the Current Classifications and Radiographic Measurements for Trochlear Dysplasia Appropriate in the Skeletally Immature Patient? Orthop. J. Sport. Med. 4:1-10

Tscholl PM, Wanivenhaus F, Fucentese SF (2017) Conventional Radiographs and Magnetic Resonance Imaging for the Analysis of Trochlear Dysplasia The Influence of Selected Levels on Magnetic Resonance Imaging. Am J Sport Med 45:1059-1065

A biomechanical study demonstrated that the Dejour classification might be relevant in respect to patellofemorale pressure aspects, but not in respect to stability [19]. That means that the trochlear bump can increase PF pressure and thereby it be related to AKP, however in the most prevailing use of the Dejour classification, namely in relation to patellar instability, there is minor relevance. Several have given different suggestions for TD classifications, however one that is based upon biomechanical and objective findings, and not subjective perceptions, is still absent $[7,20]$. In a review focusing measurements that characterize TD, the most important objective measurement was found to be the lateral trochlea inclination angle (LTI) [21]. The LTI angle express the proportion of lateral osseous support for the patella [22], and this is supported by simple logic and also a computational study [23]. Since trochlear dysplasia is localized proximal in the groove, it is crucial to measure LTI in one of the most proximal MRI axial slices.

Frequently is the term patella tilt misinterpret. It is right that the patella can tilt laterally, however in most situations this is secondary to a TD configuration having a low LTI. When TD is present you will first of all typically identify a patella with a short medial facet and secondly observe that only the lateral patella facet articulates with the flat trochlea. With this said there are cases with patella tilt due to a tight lateral retinaculum. It is important to separate those two types of origins. If you have a Lateral Patellar Compression Syndrome with the classic movie sign symptom, you can expect a positive outcome by doing a lateral lengthening, however if the tilt is caused by TD, there will be an increased risk of medial patella subluxations [25].

\section{Trochleoplasty}

Trochleoplasty is today a well-established procedure, however despite this are there still many who are skeptical. There are still many unanswered questions, but also a number of studies demonstrating favorable outcomes [25-27]. A frequent critical question is the concerns in respect to development of osteoarthritis $(\mathrm{OA})$ ? The answer is we don't know, but data implicate that there is not increased risk [28]. In fact, it is well known that both TD and recurrent patellar instabilities both increases the risk of $\mathrm{OA}$, and there the number of biomechanical and clinical observations that points in the direction that the realignment even protect against further development of OA. It is claimed that Trochleoplasty is a risky procedure, but in a study from Van Sambeeck et al. [29], the rate of complications was found similar to other patella stabilizing procedures. Next is if the patella fit the new trochlea. The short answer is yes, however in some rare cases, with a very concave lateral facet of the patella, it will be a problem. Fucentese et al. [30] investigated patellar morphology in knees having TD, and the key morphologic change of the patella, was a decreased medial facet length. This means that when the lateral trochlea inclination angle is normalized after a correctly performed Trochleoplasty, the patella's lateral facet will articulate with the lateral wall of the new trochlea, and correspondingly the preoperative patella tilt will be reduced. See Figure 3. 


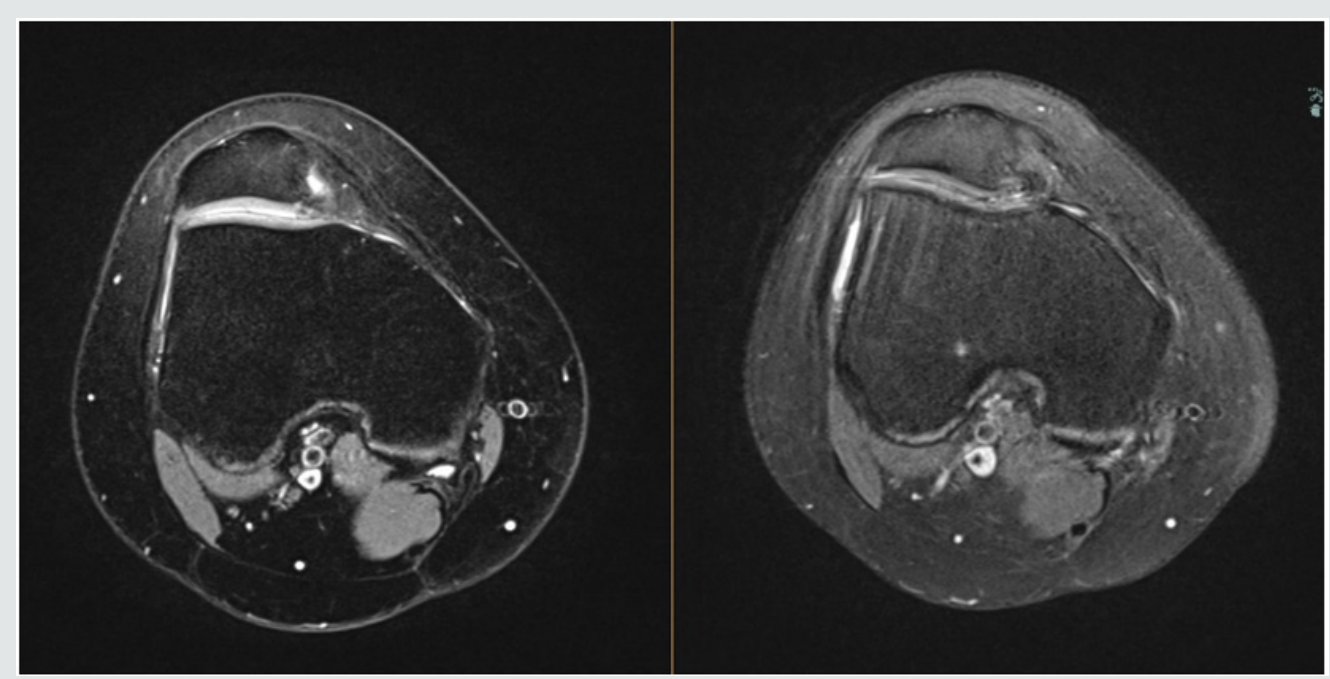

Figure 3: This is the same knee having trochlear dysplasia (left) and three years after arthroscopic Trochleoplasty. This demonstrate how the lateral trochlea inclination angle can be normalized.

\section{Clinical examination}

In respect to objectively determine the degree of instability, Zimmermann et al. [31] have recently provided us with a new examination tool. This is the reverse dynamic patella apprehension test and many surgeons have already in cooperated this as a standard evaluation tool, when deciding for a bony procedure in the treatment of patellar instability.

\section{Anterior knee pain (AKP)}

For years it has been discussed if there was a link between AKP in younger age and later patellofemoral osteoarthritis. New data indicate that there is a relation $[32,33]$. Conchie et al. reported that 7.5 times higher risk of Patellofemoral arthritis if they had AKP in adolescence.

\section{Hoffa fat pad impingement}

A frequent misinterpretation is when the term Hoffa Fat Pad Impingement is used as a diagnosis. Hoffa Fat Pad edema is a secondary result of Patella Alta and it is caused by an overload of the proximal part of the Fat Pad. This happens since the Patella is not engaged properly in the trochlear groove, and therefore the load that should have been absorbed by the patella is instead transferred to the Fat Pad instead. This Fat Pad overload is observed as edema on MRI [34-36]. Some papers have documented the relationship between having AKP, Patella alta and Fad Pad edema [11-38].

\section{References}

1. Magnussen RA, Verlage M, Stock E, Zurek L, Flanigan DC, et al. (2017) Primary patellar dislocations without surgical stabilization or recurrence: how well are these patients really doing? Knee Surg Sports Traumatol Arthrosc 25(8): 2352-2356.

2. Pagliazzi G, Napoli F, Previtali D, Filardo G, Zaffagnini PS, et al. (2019) Treatment of Primary Patella Dislocation. Arthroscopy 35(8): 24692481.

3. Migliorini F, Driessen A, Quack V, Gatz M, Tingart M, et al. (2020) Surgical versus conservative treatment for first patellofemoral dislocations: a meta - analysis of clinical trials. Eur J Orthop Surg Traumatol (0123456789).

4. Kaewkongnok B, Bøvling A, Milandt N, Møllenborg C, Viberg B, et al. (2018) Does different duration of non-operative immobilization have an effect on the redislocation rate of primary patellar dislocation? A retrospective multicenter cohort study. Knee 25(1): 51-58.

5. Tan SHS, Lim BY, Chng KSJ, Doshi C, Wong FKL, et al. (2019) The Difference between Computed Tomography and Magnetic Resonance Imaging Measurements of Tibial Tubercle-Trochlear Groove Distance for Patients with or without Patellofemoral Instability: A Systematic Review and Meta-Analysis. J Knee Surg [Internet].

6. Hingelbaum S, Best R, Huth J, Wagner D, Bauer G, et al. (2014) The TT-TG Index: a new knee size adjusted measure method to determine the TTTG distance. Knee Surg Sports Traumatol Arthrosc 22(10): 2388-2395.

7. Blønd L (2017) Patellar instability - An update. Acta Orthop Belg [Internet] 83(3): 367-386.

8. Biedert RM, Albrecht S (2006) The patellotrochlear index: a new index for assessing patellar height. Knee Surg Sports Traumatol Arthrosc 14(8):707-712.

9. Dejour D, Ferrua P, Ntagiopoulos PG, Radier C, Hulet C, et al. (2013) The introduction of a new MRI index to evaluate sagittal patellofemoral engagement. Orthop Traumatol Surg Res [Internet] 99(8): 391-398.

10. Ahmad M, Janardhan S, Amerasekera S, Nightingale P, Ashraf T, et al. (2019) Reliability of patellotrochlear index in patellar height assessment on MRI - correction for variation due to change in knee flexion. Skeletal Radiol 48(3): 387-393.

11. Luyckx T, Didden K, Vandenneucker H, Labey L, Innocenti B, et al. (2009) Is there a biomechanical explanation for anterior knee pain in patients with patella alta? : influence of patellar height on patellofemoral contact force, contact area and contact pressure. J Bone Joint Surg Br 91(3): 344350 .

12. Gravesen KS, Kallemose T, Blønd L, Troelsen A, Barfod KW (2019) Persistent morbidity after Medial Patellofemoral Ligament Reconstruction - A registry study with an eight-year follow-up on a nationwide cohort from 1996 to 2014. Knee 26(1): 20-25.

13. Hiemstra LA, Kerslake S, Loewen M, Lafave M (2016) Effect of trochlear dysplasia on outcomes after isolated soft tissue stabilization for patellar instability. Am J Sports Med 44(6): 1515-1523. 
14. Balcarek P, Rehn S, Howells NR, Eldridge JD, Kita K, et al. (2016) Results of medial patellofemoral ligament reconstruction compared with trochleoplasty plus individual extensor apparatus balancing in patellar instability caused by severe trochlear dysplasia : a systematic review and meta - analysis. Knee Surg Sports Traumatol Arthrosc 25(12): 38693877.

15. Lippacher S, Dejour D, Elsharkawi M, Dornacher D, Ring C, et al. (2012) Observer Agreement on the Dejour Trochlear Dysplasia Classification: A Comparison of True Lateral Radiographs and Axial Magnetic Resonance Images. Am J Sport Med 40(4): 837-843.

16. Nelitz M, Lippacher S, Reichel H, Dornacher D (2014) Evaluation of trochlear dysplasia using MRI: correlation between the classification system of Dejour and objective parameters of trochlear dysplasia. Knee Surg Sports Traumatol Arthrosc 22(1): 120-127.

17. Mousinho R de SMS, Ribeiro JNA, Pedrosa FKS, Lima DA de, Gonçalves RK, et al. (2019) Avaliação da reprodutibilidade da classificação de Dejour para instabilidade femoropatelar. Revi Brasi Ortop 54(2): 171177.

18. Brady JM, Sullivan JP, Nguyen J, Mintz D, Green DW, et al. (2017) The Tibial Tubercle-to-Trochlear Groove Distance Is Reliable in the Setting of Trochlear Dysplasia, and Superior to the Tibial Tubercle-to-Posterior Cruciate Ligament Distance When Evaluating Coronal Malalignment in Patellofemoral Instability. Arthrosc - J Arthrosc Relat Surg 33(11): 20262034.

19. Van Haver A, De Roo K, De Beule M, Labey L, De Baets P, et al. (2015) The Effect of Trochlear Dysplasia on Patellofemoral Biomechanics: A Cadaveric Study with Simulated Trochlear Deformities. Am J Sport Med 43 (6): 1354-1361

20. Sharma N, Brown A, Bouras T, Kuiper JH, Eldridge J, et al. (2020) The Oswestry-Bristol Classification. Bone Joint J 102(1): 102-107.

21. Paiva M, Blønd L, Hölmich P, Steensen RN, Diederichs G, et al. (2018) Quality assessment of radiological measurements of trochlear dysplasia; a literature review. Knee Surgery Sport Traumatol Arthrosc 26(3): 746755.

22. Carrillon Y, Abidi H, Dejour D, Fantino O, Moyen B, et al. (2000) Patellar instability: assessment on MR images by measuring the lateral trochlear inclination-initial experience. Radiology 216(2): 582-585.

23. Rezvanifar SC, Flesher BL, Jones KC, Elias JJ (2019) The Knee Lateral patellar maltracking due to trochlear dysplasia : A computational study. Knee 26(6): 1234-1242.

24. Pagenstert G, Wolf N, Bachmann M, Gravius S, Barg A, et al. (2012) Open lateral patellar retinacular lengthening versus open retinacular release in lateral patellar hypercompression syndrome: A prospective doubleblinded comparative study on complications and outcome. Arthroscopy 28(6): 788-797.

25. Hiemstra LA, Peterson D, Youssef M, Soliman J, Banfield L, et al. (2019) Trochleoplasty provides good clinical outcomes and an acceptable complication profile in both short and long-term follow-up. Knee Surg Sports Traumatol Arthrosc 27(9): 2967-2983.

26. Longo UG, Vincenzo C, Mannering N, Ciuffreda M, Salvatore G, et al. (2018) Trochleoplasty techniques provide good clinical results in patients with trochlear dysplasia. Knee Surg Sports Traumatol Arthrosc 26(9): 2640-2658.

27. Wind RJP, Heesterbeek PJC, Wymenga AB (2018) A combined procedure with Bereiter-type trochleoplasty leads to a stable patellofemoral joint at 5-year follow-up. Knee Surgery Sport Traumatol Arthrosc 27(3): 1-8.

28. Metcalfe AJ, Clark DA, Kemp MA, Eldridge JD (2017) Trochleoplasty with a flexible osteochondral flap. Bone Joint J 99(3): 344-350.

29. van Sambeeck JDP, van de Groes SAW, Verdonschot N, Hannink G (2018) Trochleoplasty procedures show complication rates similar to other patellar-stabilizing procedures. Knee Surg Sports Traumatol Arthrosc 26(9): 2841-2857.

30. Fucentese SF, von Roll A, Koch PP, Epari DR, Fuchs B, et al. (2006) The patella morphology in trochlear dysplasia-a comparative MRI study. Knee 13(2): 145-150.

31. Zimmermann F, Liebensteiner MC, Balcarek P (2019) The reversed dynamic patellar apprehension test mimics anatomical complexity in lateral patellar instability. Knee Surg Sports Traumatol Arthrosc 27(2): 604-610.

32. Hunter D, Zhang Y, Niu J, Felson M, Kwoh KMD, et al. (2007) Patella malalignment, pain and patellofemoral progression: The Health. Osteoarthr Cartil 15(10): 1120-1127.

33. Conchie H, Clark D, Metcalfe A, Eldridge J, Whitehouse M (2016) Adolescent knee pain and patellar dislocations are associated with patellofemoral osteoarthritis in adulthood: A case control study. Knee 23(4): 708-711.

34. Matcuk GR, Cen SY, Keyfes V, Patel DB, Gottsegen CJ, et al. (2014) Superolateral Hoffa fat-pad edema and patellofemoral maltracking: Predictive modeling. Am J Roentgenol 203(2): 207-212.

35. Tsavalas N, Karantanas AH (2013) Suprapatellar fat-pad mass effect: Mri findings and correlation with anterior knee pain. Am J Roentgenol 200(3): 291-296.

36. Widjajahakim R, Roux M, Jarraya M, Roemer FW, Neogi T, et al. (2017) Relationship of Trochlear Morphology and Patellofemoral Joint Alignment to Superolateral Hoffa Fat Pad Edema on MR Images in Individuals with or at Risk for Osteoarthritis of the Knee: The MOST Study. Radiology 284(3): 806-814.

37. Blønd L (2018) Does the patellofemoral joint need articular cartilage ? clinical relevance. Ann Jt 3(5):1-6.

38. Jarraya M, Diaz LE, Roemer FW, Arndt WF, Goud AR, et al. (2018) MRI Findings Consistent with Peripatellar Fat Pad Impingement: How Much Related to Patellofemoral Maltracking ? Magn Reson Med Sci 17(3): 195202.

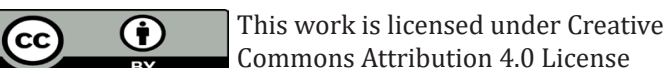

To Submit Your Article Click Here:
Submit Article
DOI: 10.32474/OSMOAJ.2020.03.000171

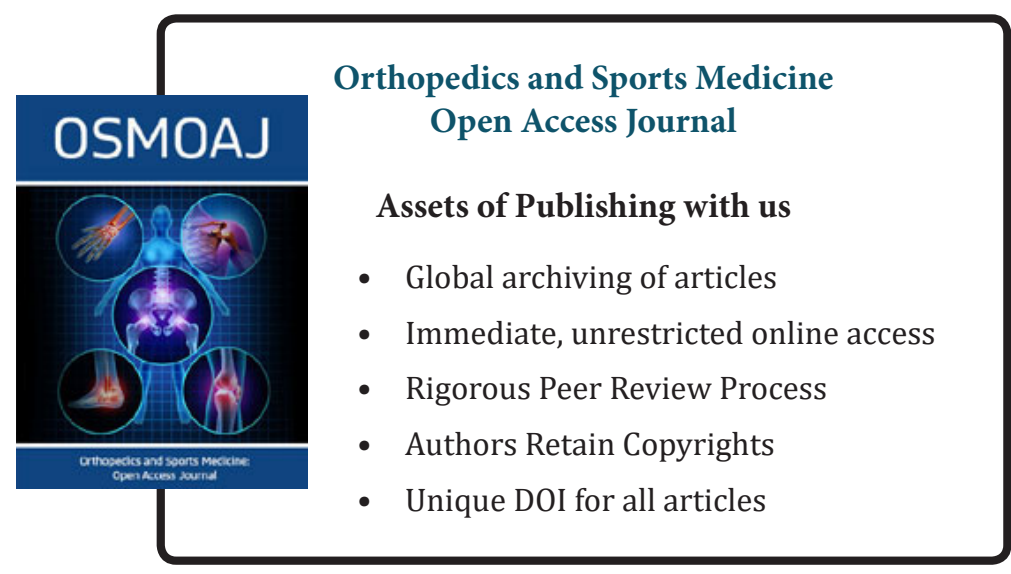

